\title{
El servicio de referencia digital
}

Por Purificación García Delgado

Resumen: Partiendo una somera definición del servicio de referencia digital y de algunas recomendaciones en cuanto a criterios de calidad y normalización, se analizan las tecnologías actuales más importantes en referencia digital desde el correo electrónico hasta el software de contacto web, que integra, entre otros, correo electrónico, chat, $M O O$, videoconferencia y navegación compartida, así como los pro-

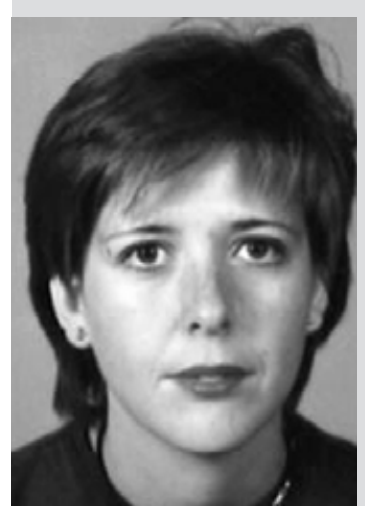
yectos más importantes realizados hasta la fecha en EUA y Reino Unido. Finalmente se comentan experiencias en biblioPurificación García Delgado tecas españolas.

Palabras clave: Servicio de referencia digital, Correo electrónico, Chat, Videoconferencia, $\mathrm{MOO}, \mathrm{Na}$ vegación compartida, Cdrs, VRD.

\section{Title: Digital reference ser-} vices

Abstract: This article analyses digital reference services and offers definitions of the concept as well as recommendations about quality criteria and standardisation. A discussion follows covering the most important technologies from e-mail to web contact software that integrates e-mail, chat, MOO's, videoconferencing and web co-navigation together with other functionality. The author describes the main projects developed in this area in the US and the UK, as well as specific experiences from Spain.

Keywords: Digital reference services, E-mail, Chat, Videoconferencing, MOO's, Web co-navigation, Cdrs, VRD 
LA MAYORÍA DE LOS AUTORES están de acuerdo en que el servicio de referencia virtual está basado más en la forma que en el fondo, es decir, que toda la transacción de referencia es llevada a cabo de modo virtual siguiendo la máxima de Taylor "en cualquier sitio, espacio, a cualquier ritmo y en cualquier momento". Aquí radica lo novedoso de este servicio: se ofrece total libertad de acceso al mismo.

En esta línea se expresan autores como Marylin D. White quien

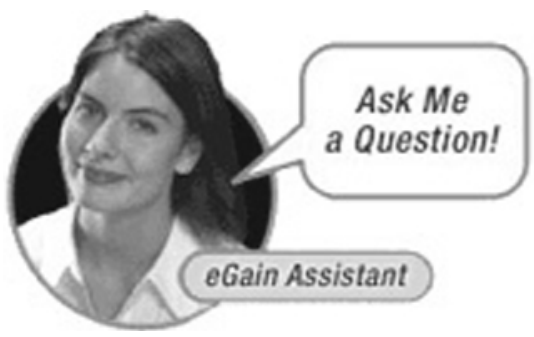

lo entiende como un servicio de acceso de información a través de medios electrónicos. Otras definiciones que versan sobre las mismas características las tenemos en las definiciones del VRD (Virtual Referente Desk) y Wasik.

\section{Ejemplo de funcionamiento de web contact center software}

Una empresaria busca información de cómo comenzar un negocio. No quiere desplazarse a la biblioteca y desde casa accede a su página web, donde encuentra un icono que dice "Pinche aquí para hablar con un bibliotecario". Así lo hace y a continuación se le ofrece la posibilidad de enviar un correo electrónico, abrir una sesión de chat o que el bibliotecario le devuelva la llamada. Elige chatear.

Se le pide información que la identifique: su nombre y dirección de correo, número de carné de biblioteca, etc. Una vez ya conectada recibe un mensaje de bienvenida. Si el bibliotecario está ocupado se le ofrecerá la posibilidad de conocer mejor otros servicios, es lo que se denomina "páginas de espera" (pages on hold), de manera que la biblioteca le puede ofrecer un conjunto de urls o información que envía directamente a su navegador mientras espera. En otros casos también se ofrece hilo musical.

El software redirecciona a una cola la petición recibida, de acuerdo con las normas que la biblioteca haya diseñado y brinda también al bibliotecario la posibilidad de recuperar mensajes ya creados para responder directamente a preguntas generales. Este mensaje preconfigurado ha de contener siempre fórmulas de cortesía y ha de acabar preguntando si necesita más información.

Dado que la pregunta que la empresaria ha formulado es más específica, se le responde que busque en una base de datos pertinente, con lo que el bibliotecario comienza así una sesión de navegación compartida, o control remoto. El profesional manejará la pantalla y el ratón de la usuaria, mostrándole los pasos a seguir y, si tiene dificultades a la hora de realizar la búsqueda en la base de datos, el bibliotecario puede elegir la posibilidad de formulario compartido (form share) de manera que vea qué términos está introduciendo ella.

Una vez satisfecha la necesidad de información, el sistema enviará automáticamente a la usuaria una trascripción de toda la consulta, con la conversación mantenida en el chat al completo y las urls, así como una copia a la biblioteca para su archivo y análisis estadístico. El software también genera informes con datos como el número total de sesiones, tiempo medio de respuesta, preguntas por área temática, momentos punta del servicio, etc.

A mi entender, Lankes, en su definición de "interfaces que median entre el usuario en un 'anómalo' estado de conocimiento y una colección de información” está restringiendo demasiado las posibilidades de dicho servicio pues se desestiman sistemas como el chateo en voz en directo o la videoconferencia.

«Existen 3 ca-
racterísticas in-
trínsecas y pe-
culiares del ser-
vicio de referen-
cia a tener en
cuenta a la hora
de implementar-
lo: la escalabili-
dad, la ambigüe-
dad y el tiempo
de respuesta»

Existen 3 características intrínsecas y peculiares del servicio de referencia a tener en cuenta a la hora de implementarlo: la escalabilidad, la ambigüedad y el tiempo de respuesta. La primera se refiere a la cantidad de solicitudes que el servicio puede responder de acuerdo con los criterios de calidad marcados. En algunos ejemplos que nos podemos encontrar en EUA la simple inclusión de un botón ofertando el servicio hizo que la demanda se triplicara en escaso tiempo y se colapsara, quedando inhabilitado. Además, claro está, hay que considerar las "complicaciones" para diseñar un servicio de funcionamiento 24/7. Los ya en marcha hoy día han tenido dos opciones: aumentar la plantilla de personal o configurar un servicio de referencia cooperativo con bibliotecas de diferentes zonas horarias, como es el caso del Cdrs.

La ambigüedad se solventará cuando el equipo de referencia sepa determinar la cantidad de información apropiada para el usuario, su aplicabilidad y el nivel de infor- 
mación requerido, tal y como señala Wasik. En cuanto al tiempo, si la respuesta es inmediata entonces estamos ante un servicio de referencia digital instantáneo y su planificación e implementación es bastante diferente de otro "en diferido" que puede llevar desde un día laborable a meses la elaboración y envío de la respuesta.

Podríamos preguntarnos también por la viabilidad económica. En este sentido, Kelly Broughton señala que es totalmente lógico que este tipo de servicio se implemente pues se gastan cientos de miles de US\$ en hacer que los recursos sean accesibles de manera remota y ahora necesitamos servir a la gente que los utiliza. Además, añade, si no lo hacemos los bibliotecarios alguien más lo hará. Productos como Questia o XanEdu que se ofertan a la comunidad universitaria dan la falsa impresión de que una investigación puede ser fácil. Además, ofrecen el contenido que ya la biblioteca ha comprado por otro lado.

En definitiva, vemos que lo claramente definitorio del concepto es la tecnología aplicada que hoy día posibilita una respuesta inmediata a todo usuario, en cualquier sitio y momento. Esto, obviamente, redunda en una nueva concepción del trabajo de referencia en la biblioteca como veremos más adelante.

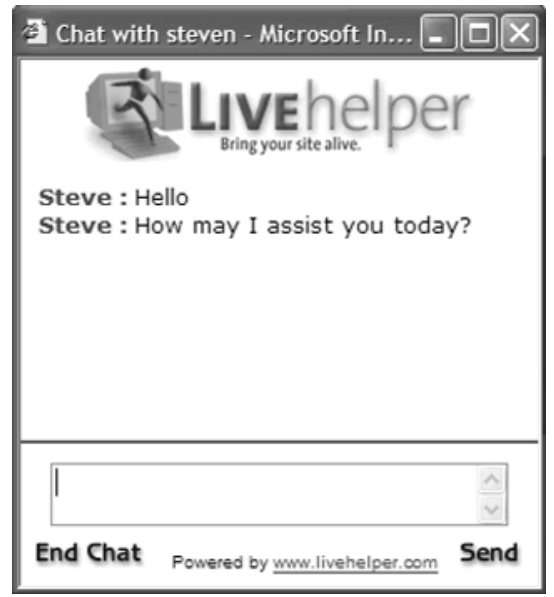

Por tanto, dado que gracias a la tecnología virtual podemos obviar el cuándo (24/7) y el dónde (desde cualquier conexión de internet), vamos a pasar a ver qué debe ofrecer el servicio de referencia, cómo se puede hacer (tecnologías disponibles) y por quiénes.

\section{Qué debe ofrecer el servicio de referencia}

Katz señala que diversos estudios indican que la mayoría de las respuestas que buscan los investigadores viene de la mano de los "colegios invisibles".

La primera y posiblemente única fuente de información es una conversación con un amigo conocedor de la materia, si no tiene esa posibilidad acudirá a internet; un tercer recurso es su biblioteca personal. Finalmente si ninguna de estas opciones le resuelve la duda, acudirá a la biblioteca. En este caso no nos podemos permitir el "lujo" de frustrar la obtención de información por parte del usuario. Es un comienzo para empezar a invertir esta cadena, especialmente en el momento en que el usuario prefiere acudir a internet por sí solo que ir a un servicio especializado.

Lankes identifica cinco componentes fundamentales en los servicios de referencia digital:

-Recepción por vía electrónica de la pregunta.

—Envío al especialista, puede que la pregunta pase a una cola de espera de acuerdo con los criterios marcados por el servicio.

- Composición de la respuesta de acuerdo con la política del servicio.

—Envío de la respuesta.

- Seguimiento e identificación de los temas más demandados para estadísticas y generación de archivos.

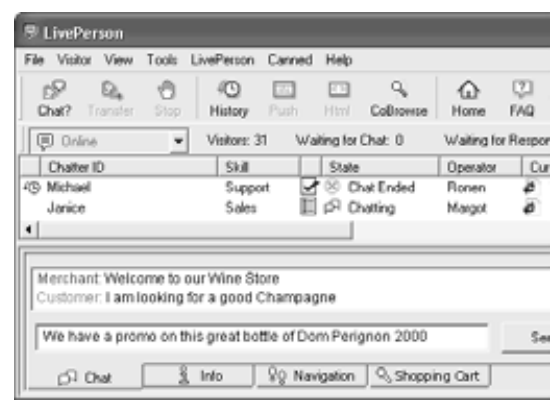

El servicio de referencia digital se acerca más a las necesidades del usuario en tanto que elimina barreras de lugar y espacio, pero la entrevista de referencia en que se basa se complica un poco al no haber interacción cara a cara.

En este sentido señalamos algunos de los criterios de calidad de Bennet, Kasowitz y Lankes, obtenidos del proyecto $K-12$ AskA Service. La entrevista de referencia virtual ha de ser especialmente cuidadosa con la privacidad del usuario y recomiendan que aparezcan las políticas al respecto en la página web del servicio. En las respuestas se ha de procurar incluir información relacionada y el proceso de consulta de las diferentes fuentes así como ofrecer los métodos para encontrar los mejores recursos. Finalmente se ha de ser muy claro con el tipo de información que el usuario puede obtener para reducir las oportunidades de confusión y expectativas por su parte.

De especial interés resulta el modelo de respuesta del $V R D$, a través del cual se consigue una uniformidad en la calidad del servicio y superar algunas de las barreras de la entrevista de referencia digital. Así, cualquier respuesta obtenida de su servicio de referencia contendrá lo siguiente:

—La definición de la tarea: identifica la cuestión y las características del usuario para así determinar el tipo apropiado de respuesta. No recibirá el mismo tipo de información un profesor de química que un niño en edad escolar. 
— La/s estrategia/s de búsqueda de información: han de ser comunicadas al usuario de manera que, como "valor añadido", se le está formando para que en otra ocasión pueda funcionar de forma autónoma.

\section{«El modelo de respuesta del VRD consigue una uniformidad en la calidad del servicio y supe- rar algunas de las barreras de la entrevista de re- ferencia digital»}

- La localización y acceso de las fuentes seleccionadas: se le comunica dónde se encuentran los recursos en los que se ha basado la respuesta y su tipo de acceso; por ejemplo, si se ha extraído de una publicación electrónica, si necesita de contraseña, etc.

- Toda respuesta ha de asesorar sobre la información hallada, evaluándola y recomendado su mejor uso. También ha de sintetizarse utilizando lenguaje claro y apropiado según el usuario que se ha identificado en una primera instancia.

-Por último, antes de enviarla, el experto evaluará todo el contenido así como el tono y el lenguaje empleado en el mensaje.

\section{Cómo se puede ofrecer el servicio de referencia digital: tecnologías aplicables}

A continuación reseñamos, de la más antigua (correo electrónico) a la más reciente (web contact software), las técnicas utilizadas. En cuanto a servicio en sí, uno de los primeros fue Electronic Access to Reference Service (Ears) de la biblioteca de Ciencias de la Salud de la Universidad de Maryland, lan- zado en 1984; otros ejemplos son AskEric, de 1992, y la Internet Public Library de 1995.

\section{Correo electrónico y Use- net.}

Envía las preguntas a la biblioteca. Es el método elegido para grupos de noticias y grupos de discusión con Listserv. Un tipo similar de grupos de discusión es Usenet.

Algunas listas de distribución a veces se denominan también Listservs, tomando el nombre del software de gestión de la lista, normalmente usado en Bitnet. Listserv es más que un software de gestión de la lista, en realidad es una red de ellas funcionando en sistemas que están unidos y que pueden procesar comandos compartidos. Por ejemplo, si se envía el comando "list global" a un máquina Listserv, recuperará el total de listas de distribución Bitnet. Otras aplicaciones de gestión de correo electrónico pueden ser Majordomo y Listproc.

Para los profesionales es de gran interés Stumpers, la lista de distribución para los bibliotecarios referencistas, especialmente para preguntas difíciles de referencia; Internet Hunt es similar. Podemos emplear también New-list, una lista de distribución que informa de nuevos grupos de correo, disponible en Usenet como bit.listserv.new-list. Otras de contenido afín son: $B L-L$ para bibliografía y LibRef-L para referencia general. Dig_Ref es la lista para el servicio de referencia virtual, creada en 1998 y su meta es poner en contacto a bibliotecarios de referencia virtual de manera que puedan comentar cualquier aspecto relacionado con este tipo de servicio, desde cómo se crea hasta criterios de calidad, etc.

http://www.vrd.org/Dig_Refldig_re f.shtml

Existen también directorios en línea de grupos de noticias. Por ejemplo, si nos conectamos al
Gopher de la Universidad de Baylor tiene la base de datos de Bitnet/Internet E-mail Groups. También el Directory of Scholarly Electronic Conferences, creado y mantenido por un equipo en la Universidad Estatal de Kent. http://www.mid.net/KOVACS

Algunos Usenet que pueden ser útiles para los bibliotecarios son los siguientes:

-Alt.Quotations. Para preguntar sobre el origen de una cita cualquiera. No tiene moderador pero es de gran utilidad para recuperar una referencia o para compartir tu cita favorita.

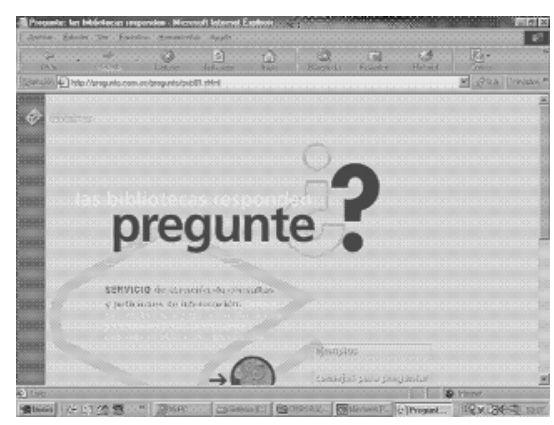

http://wilma.cs.brown.edu:/pub/alt .quotations/FAQ.txt

-Rec.Arts.Books Dedicada a la crítica o evaluaciones de libros por parte de expertos. Para materiales específicos como películas o música están Rec.Arts.Movies y Rec.Music.

http://danny.oz.au/books/rabr/

Según Bernie Sloan, el modelo o escenario de referencia para este tipo de servicio ha de utilizar formularios web con los datos que contiene el modelo aportado por Abels: personales, materia y restricciones del proceso de búsqueda como idioma, fechas, etc. Se le debe indicar al usuario el tiempo medio de espera para recibir respuesta y, para evitar una masiva llegada de preguntas, limitar el servicio a aquellos pertenecientes a la institución y, si es necesario, al tipo de preguntas. 
Abels señala que tiene sus ventajas y desventajas. Entre las primeras nos encontramos con que es un método fácil de comunicar, los mensajes son cortos, es barato, rápido y la demanda puede hacerse fuera de las horas de apertura de la biblioteca. Como desventaja principal está que es un procedimiento asíncrono. Según la misma autora, la entrevista de referencia ideal está compuesta por 3 correos electrónicos: en el primero el usuario envía la pregunta mediante un formulario, pues éste es el mejor método; el segundo es el envío de la respuesta y el tercero la confirmación del usuario. Si fuera necesario continuar el diálogo Abels aconseja pasar a otro tipo de comunicación.

\section{Software de chat.}

Una de sus grandes ventajas es que es gratuito. Algunos ejemplos son: Netscape Instant Messenger, HumanClick (permite ayuda en tiempo real) y Livehelper. Alguna universidad incluso se ha atrevido a desarrollar su propio software, como el caso de la Universidad de Temple, con Camden o el Morris Messenger de la Universidad del Sur de Illinois (gratuito). http://www.humanclick.com http://www.livehelper.com http://nimbus.oci.temple.edu/ jlab onsk/camden.html

La Universidad del Norte de Texas eligió el software WebMaster ConferenceRoom Personal Edition porque, aparte del precio (99 US\$), era líder en el mercado, ofrecía un entorno $I R C$ que el sistema de la biblioteca era capaz de soportar y finalmente, ofrecía 3 importantísimas características: privacidad en la entrevista con el usuario, posibilidad de echarlos si su comportamiento era inadecuado y era viable la grabación de las transacciones.

http://www.webmaster.com

a. Cómo funciona para el bibliotecario. El profesional abre el

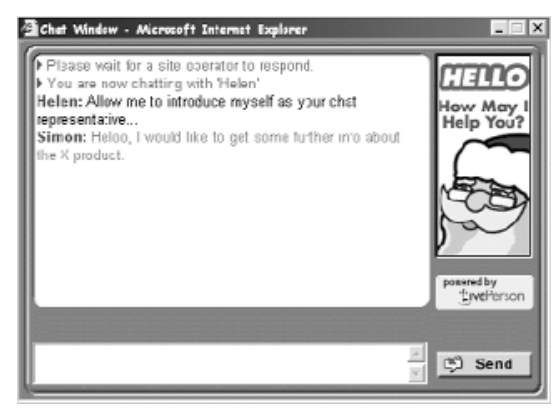

programa cliente de IRC, Xircon. Una vez conectado al servidor, debe unirse a la sesión de chat. En el caso del software WebMaster ConferenceRoom lo hace tecleando lo siguiente /join\#helpdesk, siendo helpdesk el nombre del canal para chatear.

A continuación le aparece una lista de personas (usuarios y bibliotecarios) que están conectados. Si el nombre está precedido por @, significa que es un operador, que es la primera persona que se conecta al sistema y que tiene categoría de administrador. Puede: seleccionar un tema para el canal, echar a usuarios no deseados, prohibir a uno utilizar el canal, cambiar las propiedades como número de personas que pueden estar conectadas, canales privados o secretos, etc. También tiene la capacidad de filtrar palabras, como palabrotas, de manera que si el usuario las introduce el mensaje no se envía y se le comunica que ha de reformular su pregunta.

Existen 3 tipos de tecnologías chat: envío instantáneo de mensajes (instant messaging software), software de chat y chat rooms. El primero requiere que el programa esté instalado en todos los ordenadores y ofrece como principales ventajas que es gratuito, fácil de instalar y no necesita un ancho de banda especial. Algunos ejemplos son: $A O L$ Instant Messenger, ICQ de Mirabilis Ltd., Microsoft Messenger o Yahoo! Messenger.

En cuanto a los chat rooms son menos atractivos y requiere que la biblioteca se registre en un sitio web de una compañía que ofrezca dicho servicio. Ejemplos son: Anexa, ChatSpace o Yahoo!Clubs.

WebChat es un software gratuito disponible para Unix, Windows95 y Windows NT. Es más primitivo que $I R C$ pero sólo requiere un navegador para acceder.

El Florida Distance Learning Reference \& Referral Center $(R R C)$ aconseja tomar en consideración las siguientes cuestiones a la hora de escoger un sistema u otro: su coste, que sea compatible con el servidor de la institución, que se pueda testar primero a través de una demo y que tenga funcionalidades como: chateo con moderador, filtros, posibilidad de prohibir a determinados usuarios, registro de las sesiones para su posterior tratamiento estadístico, opción de ofrecer manuales en línea y de personalizar las interfaces y que, finalmente, no requiera ningún coste al usuario ni un tipo específico de software. Como ejemplo de servicio podemos indicar InfoChat, de las Auburn University Libraries, que utiliza HumanClick; en un semestre consiguieron como media semanal 9.6 preguntas a través de chat y 7.4 sesiones de chat por semana.

\section{MOO's (Multi-User Object Oriented).}

Originalmente se desarrollaron para juegos interactivos en tiempo real en internet y proporcionan un entorno virtual multiusuario así como enlaces comentados a muchos sitios de interés. No es más que una gran base de datos en la que reside un servidor enlazado a internet, ofrece un lugar para conversación en directo y se parece mucho a un chat, aunque supera a éste en prestaciones. Por ejemplo, permite crear y programar objetos individuales en el $M O O$ para que otros interactúen con él.

Sus principales ventajas son que no necesita mucho ancho de 
banda y que el usuario no tiene por qué tener la última tecnología. En cuanto a las desventajas, decir que está limitado a una interfaz basada en texto. Los jugadores o "actores" se organizan jerárquicamente, según su experiencia o habilidad, mientras que los nuevos comienzan como constructores o programadores, antes de convertirse en "wizards". Desde un punto de vista de programación, los objetos $M O O$ se definen por una serie de verbos o procedimientos y propiedades o variables.

Un ejemplo es la biblioteca de la Universidad de New Brunswick, que lo emplea para los estudiantes a distancia. En cuanto al software podemos reseñar Diversity University $\mathrm{MOO}$ o CoMentor.

http://www.unb.ca:7000/

http://www.lib.unb.ca/Services/Dis tEd/moo_du_org_8000.html

http://comentor.hud.ac.uk/welcom e.htm

A modo de ejemplo, comentaremos el proyecto ArkMOO de la Universidad de Arkansas que integra $M O O$ con internet y correo electrónico. El usuario puede acceder al sistema a través de un cliente telnet basado en Java y a partir de ahí pide determinadas páginas que aparecen en una ventana de navegador. Se organizó un espacio para biblioteca y después diferentes áreas para cada curso.

\section{Experiencias en videocon- ferencias.}

La videoconferencia es la tecnología que más se aproxima a una entrevista de referencia tradicional pues existe contacto visual en tiempo real. En este caso destacamos dos proyectos estadounidenses llevados a cabo con esta tecnología: See You See a Librarian e Interactive Reference Asístanse (IRA) de la Universidad de Michigan con el software CU-SeeMe. Sin embargo, resultó que tanto el equipo físico como lógico no esta-

ban lo suficientemente desarrollado y los bibliotecarios se encontraron con poco apoyo informático. $\mathrm{Cu}$-SeeMe es un software gratuito de videoconferencia que proporciona imagen y sonido. Todas las máquinas que participen han de tener tarjetas de vídeo/audio y además consume mucho ancho de banda.

\section{«La entrevista de referencia vir- tual ha de ser especialmente cuidadosa con la privacidad del usuario y se re- comienda que aparezcan las políticas al res- pecto en la pági- na web del ser- vicio»}

Por su parte, Interactive Reference Service (IRS) de la Universidad de California, Irvine, los usuarios se quejaron de que al final tenían que seguir yendo a la biblioteca a recoger el material y sugirieron que las sesiones se llevaran a cabo en una habitación aparte. Los bibliotecarios pidieron monitores más grandes y una alerta auditiva para avisarles de que un usuario necesitaba ayuda.

Las tecnologías de videoconferencia necesitan de determinada infraestructura que puede no representar un problema para la biblioteca pero sí para el usuario remoto. Otra de las principales desventajas se produce cuando más de dos usuarios se intentan conectar, puesto que no se ofrece un mecanismo de cola o redirección.

Estos paquetes proporcionan una conexión audio y vídeo entre ordenadores en una de las 4 formas siguientes: en área de red local (LAN), vía internet, por líneas de teléfono regulares o bien vía $I s d n$
(Integrated Services Digital Network), una línea especial digital de teléfono. Los dos primeros dependen demasiado del ancho de banda y en los momentos más descongestionados transmiten imágenes de vídeo a 15 fps.

Bernie Sloan sugiere el siguiente modelo o escenario de referencia en un servicio de vídeo conferencia: limitar el número de sitios desde los que se puede iniciar las sesiones de consulta, debido a que requiere de plantilla disponible en el momento. Por la misma razón, aduce que hay que poner un horario establecido y en el caso de que las necesidades de información no sean satisfechas, fijar hora para otro día o bien decidir cambiar a otro tipo de medio como puede ser el correo electrónico. Finalmente, conforme se vaya consolidando el servicio, planificar el número de sitios desde los que se accede.

\section{Web contact center soft- ware.}

Esta tecnología se ha extraído del mundo de venta telefónica o vía web y se está aplicando ya al servicio de referencia virtual. A la hora de recibir consultas, realiza la misma función que una centralita telefónica pero también añade nuevas posibilidades que le proporciona la web.

En lugar de enviar al usuario a un menú automático de voz se le pide que busque en el sitio web. Si todavía necesita ayuda, entonces se pone en funcionamiento el sistema de distribución automático de llamadas. El software puede ser configurado para que permita una combinación de correo electrónico, chat, devolución de llamadas, voz en IP o incluso videoconferencia. Otras ventajas añadidas son la tecnología push o de entrega de información al usuario, formularios web y control remoto del navegador del usuario (ver ejemplo adjunto). 
Steve Coffman señala algunas mejoras que deben realizarse en este tipo de software. Por un lado, las páginas de espera no son buenas pues inicialmente estas aplicaciones fueron diseñadas para empresas que vendían sus productos por internet y cuya respuesta era prácticamente inmediata. También debe mejorarse la navegación compartida, ya que los sistemas deberían escoltar al usuario por cualquier página web, incluso por las bases de datos propiedad de la biblioteca. Desafortunadamente, estos productos únicamente funcionan para pocas bases de datos. La mejor solución a este problema parece ser la navegación compartida basada en servidores proxy, como el que usa el software Lssi Interact y Cisco Dynamic Content Adapter. Sería deseable también poder incluir un botón de ayuda de referencia virtual en las propias bases de datos. Hay que considerar también la posibilidad que tienen las bibliotecas de ofrecer este tipo de servicio a otras, previo pago de una cuota.

La licencia de determinados productos también puede afectar al servicio de referencia digital. Licencias de sólo 5 usuarios al tiempo puede considerar como tal al propio bibliotecario que hace la consulta de referencia en tiempo real. Esto sería un tema a negociar con los editores.

a. Ejemplos de web contact software

-WebLine. Comparte la información con el cliente, formularios o páginas web a la vez que mantiene una conversación o bien chatea con texto. Esto incluye características como: navegación compartida, envío bidireccional de páginas, compartir formularios web, transferencia de ficheros y, además, las conversaciones pueden ser uno a uno o uno a varios.

http://www.webline.com
En la Univ. de California Los Angeles (Ucla) se testó y se encontraron algunos problemas. Por ejemplo, había que modificar el tamaño de las ventanas para que tanto el usuario como el bibliotecario pudieran ver la información, lo que confundía mucho a los primeros. También se les solicitaba permiso para cargar applets, lo que provocó que algunos desconfiaran. Este software funciona mejor con preguntas cortas y breves que se pueden responder con un sitio web. Otra pega es el tiempo de espera mientras el bibliotecario hace la búsqueda.

-EGain. Es más fácil de usar que el anterior y utiliza Hipbone para conectar los navegadores del bibliotecario y el usuario, cuya pantalla presenta la información de manera clara ya que aparece una columna con los mensajes de chat, viendo la misma página que el bibliotecario. Si el bibliotecario no quiere que la vea, ha de abrir otra ventana. Ofrece también registro de las sesiones para informes estadísticos. Finalmente hay que resaltar que es más rápido que WebLine.

http://www.egain.com/

-LivePerson. Sus principales características son: respuestas predefinidas que en muchos casos pueden solventar la consulta sin intervención del bibliotecario, transmisión del chat a los operadores apropiados de acuerdo con las normas fijadas por cada centro, registro de todo lo que el usuario consulta, muy importante para analizar el tipo de demandas y tipo de servicio a ofrecer. De especial interés es que se le puede invitar a chatear al usuario para, de este modo, ser la propia biblioteca la que sale a su encuentro de manera activa. Otra característica interesante es su servicio de alertas, de manera que cuando determinado usuario VIP se conecte avisa al operador apropiado. Una vez acabada la consulta envía la trascripción de todas las operaciones realizadas. Finalmente emite informes en tiempo real de la efectividad del sitio web y de los operadores e informes que cuantifican el impacto de las herramientas LivePerson en la experiencia del usuario.

http://www.liveperson.com

-OnDemand. Sistema elegido para integrarse con QuestionPoint de la $L C$, Oclc y resto de bibliotecas participantes en el proyecto. Utiliza el vídeo y la voz para conferencias, así como chat, urls y contenido web compartido.

http://www.conveysystems.com/lib rary.asp

-LiveAssistance. No necesita instalación alguna por parte del usuario. Ofrece historial del chat, informes estadísticos, tecnología push para el envío de cualquier tipo de fichero o urls, respuestas predefinidas y páginas web prediseñadas para una más pronta solución, se puede también enviar el chat a otro operador cuando sea requerido y, en momentos punta, el sistema envía al usuario al primer operador que se quede libre. http://www.liveassistance.com/

-Desktop Streamming. Es un software de control remoto de navegación y pantalla del usuario, incorporando chat, envío de páginas web y ftp.

http://www.desktopstreaming.com/ ad/corp/home

-LiveHelper. Se le pide al usuario que haga clic en el botón "Livehelper" para entrar en comunicación con el operador. Características: chat, chateo de voz, tecnología push, control remoto, estadísticas de uso, mensajes predefinidos, etc.

http://www.livehelper.com/

-Docutek on Campus. Sistema de internet para todo un campus, gestión de recursos electrónicos y obtención de documentos en línea. Está compuesto de Eres, que permite el envío de documentos en 
línea, Virtual Reference Librarian. Utiliza un módulo de fax para convertir el formato papel en electrónico (pdf).

http://www.docutek.com/index.html

-RightNowLive. Ofrece chat, navegación compartida con tecnología push e informes estadísticos. http://www.rightnow.com/products llive.html

La lista parece realmente interminable: EShare, WebBoard, Webcrossing, Webchat, WebEx, PlaceWare, etc.

\section{Normalización del servicio de referencia digital.}

En abril del 2001 se creó un grupo de trabajo de Niso destinado a analizar esta cuestión. Sus principales objetivos son: determinar qué aspectos y agentes de la referencia digital se pueden beneficiar de una normalización, considerando el trabajo ya realizado como posible punto de partida.

Este comité también desarrollará el protocolo en la transacción pregunta-respuesta para el intercambio de mensajes y construirán un conjunto de metadatos que identifiquen y describan los componentes clave tanto de los datos contenidos en las preguntas y respuestas como en los datos personales y de las instituciones.

En cuanto a la tecnología, se han normalizado los procesos de recogida, almacenamiento y recuperación de las transacciones de referencia. QuIP (Question Interchange Profile) consiste en metadatos para el almacenamiento y seguimiento de las transacciones de manera consistente. Kbit (Knowledge Bit) es un formato de datos estandarizados para la gestión de las operaciones y reconoce 3 tipos de preguntas: la original, (emitida por el usuario), las que se realizan en la entrevista y la pregunta que se va a responder. Además ofrece unos campos descriptivos que per- miten la identificación de la pregunta en la base de datos: origen geográfico, idioma, derechos de autor y disponibilidad. En cuanto a las respuestas, se pueden clasificar en: corta, expandida, exhaustiva, multimedia o resumen.

\section{Proyectos de servicios de referencia digital}

Los comentados a continuación están ya seguramente superados por sus nuevas versiones pero han tenido una importancia vital, a mi entender, para el desarrollo de este tipo de servicio. De su empeño en que funcionaran nos hemos beneficiado en el sentido de que ya el servicio de referencia digital esté generalizándose cada vez más. Hay que destacar especialmente el enfoque y recursos dirigidos a los niños de 12 años, los llamados k12 services, pues claramente se está apostando por una formación a las generaciones más jóvenes como una herramienta más a su alcance.

\section{Proyecto Cdrs.}

La misión del $C d r s$ es "proporcionar servicio de referencia profesional a los investigadores en cualquier momento, en cualquier sitio a través de la red digital internacional de bibliotecas". Cada biblioteca tiene un perfil, que toma en cuenta aspectos como franjas horarias en las que puede participar, fechas no hábiles porque no se trabaje en dicha biblioteca en cuestión, materia/s en la/s que esté especializada y perfil de usuarios a los que sirve, entre otros. A través de dicho perfil, se define el nivel de relación de la biblioteca local al $C d r s$, para así saber si se ha de enviar la pregunta o no.

http://www.loc.gov/rr/digiref

Las bibliotecas participantes de la primera fase del proyecto fueron: Library of Congress, National Agricultural Library, National Library of Australia, National Library of Canada, Smithsonian
American Art, University of Texas at Austin, Cornell University, Santa Monica Public Library, Morris County Public Library y el Peninsula Library System.

En un primer momento los objetivos del proyecto eran la base de datos del perfil de cada biblioteca y los datos, normas y motores de búsqueda que soportara así como el uso de e-mail versus formularios web para el envío de las preguntas. En una segunda etapa se ampliaron el número de centros participantes, se incrementó el volumen de preguntas que el sistema podía soportar, se revisó el perfil de la base de datos y se experimentó con el software para la gestión y almacenamiento de las preguntas, y se identificaron las necesidades de plantilla.

\section{The Virtual Reference Desk.}

Esta dedicado a la investigación sobre referencia digital y financiado por el Departamento de Educación de EUA. Aparte de organizar las VRD Conferences, ha generado productos como QuestionPoint y toda una serie de AskA Services, así como aplicaciones y publicaciones.

http://www.vrd.org

\section{Lssi Virtual Referente Desk. \\ Ha dado como resultado el Vir- tual Referente Toolkit, que está compuesto a su vez de una serie de aplicaciones. Su próximo desarro- llo será el Tutor.com's Live Home- work, un servicio de tutoría digital. http://www.lssi.com/virtual}

Funciona de la siguiente manera: un usuario navega buscando información. Llega al sitio web de la biblioteca y pincha en un botón (personalizable) llamado "Obtenga ayuda en directo", con lo que se inicia una sesión de referencia virtual en la que se pide en primer lugar que se conecte a través de un formulario web (configurable). 
Los datos que tiene que introducir son nombre, dirección de correo electrónico y la pregunta.

A continuación hace clic sobre el botón de "Conexión" y su pregunta es transferida a una cola, a la espera del primer bibliotecario que esté libre. El sistema le informa del tiempo medio de espera y, si se supera, le ofrece la posibilidad de enviar un correo electrónico con la pregunta.

\section{«En abril del 2001 se creó un grupo de traba- jo de Niso desti- nado a analizar la normaliza- ción del servi- cio de referen- cia digital»}

Los bibliotecarios monitorean las colas desde una ventana de sesiones, que muestra el número de usuarios que hay aguardando y el tiempo medio de espera. Normalmente se responden por orden de llegada.

Cuando el profesional responde, el sistema lanza un navegador especial que permite que ambos trabajen en línea. Una vez conectados, mantienen un diálogo mediante chat o a través del web y pueden trabajar para localizar la información que se solicita.

Otras posibilidades que tiene el bibliotecario son: enviar páginas web al usuario, navegación en ambos sentidos o la opción de sincronización de formularios de manera que tanto uno como otro puedan rellenar datos en una búsqueda o formulario dado, enviar mensajes predefinidos, transferir la sesión a otro compañero o biblioteca y, finalmente, enviar la trascripción de la sesión al correo electrónico del usuario, además de archivarla en la base de datos del sistema para futuro análisis.

\section{Askeric.}

Servicio personalizado de internet que proporciona información sobre educación a profesores, bibliotecarios, administradores, padres, etc. Es un proyecto de Eric Clearinghouse subvencionado por el Departamento de Educación de EUA, la Universidad de Siracuse y Sun Microsystems.

http://www.askeric.org

Comenzó en 1992 y actualmente sirve a 1.000 usuarios a la semana. Se ha convertido en un sistema distribuido en el que el usuario formula la consulta, se recibe en la central y es en este punto desde donde, por una parte se le envía un mensaje para que sepa que se ha recibido y, por otro lado, envía la pregunta al centro especializado en el tema de la consulta. La respuesta va directamente de dicho centro al usuario y a un archivo.

Se ha pasado de ser una colección de marcadores a un sitio web con más de 1.000 recursos. Con el tiempo se hizo necesario crear una guía de normalización de las respuestas pues cada lugar generaba su propio formato y esto confundía a los usuarios.

Otros servicios AskA que podemos reseñar son:

-Ask the Author. Las preguntas se dirigen a los autores directamente.

http://www.ipl.org/youth/AskAutho r/AskAuthor.html

-National Museum of American Art Reference. Gestionado por un bibliotecario especialista en arte.

http://nmaa-ryder.si.edu/study/ reference-main.html

-Parents AskEric.

http://ericps.ed.uiuc.edu/npin/pask eric.html

-Ask-a-Geologist. Llevado a cabo por el Geological Survey of Canada's Earth Sciences Information Centre. http://www.nrcan.gc.caless/esic/cg i-bin/askageol_e.cgi

-Ask a Librarian. Acepta preguntas de todo tipo de público. http://www.iren.net/cfpl/forms/aal form.html

-AskLN.

http://www.att.com/learningnetwo rk/asksubmit.html

-KidsConnect.

http://www.ala.org/ICONN/AskKC .html

-AskUsQuestions.com. Proyecto llevado a cabo por la Nola Regional Library System. Han adaptado programas shareware para sus necesidades y permite tanto chatear como el envío de páginas web.

http://www.AskUsQuestions.com

\section{Madsci.}

Comenzó como un sitio web de la Escuela de Medicina de la Universidad de Washington en 1995 con 40 voluntarios y ahora mismo cuenta con más de 800 científicos voluntarios que responden preguntas que tratan de materias de astronomía a zoología. Cuenta con un archivo de más de 12.000 preguntas (recibe al mes alrededor de 3.000) y respuestas. http://www.madsci.org/

Ha desarrollado su propio software llamado Moderator que procesa las preguntas y respuestas. Además realiza tareas repetitivas de mantenimiento como formatear con hipertexto las preguntas y respuestas, archiva los ficheros y actualiza el motor de búsqueda. La estructura de personal es: 824 expertos, 35 moderadores que revisan las preguntas y respuestas, 4 administradores que mantienen el sitio y un administrador del sistema.

Las recomendaciones de Lynn Bry para desarrollar un servicio de este tipo pasan fundamentalmente por automatizar y normalizar lo más posible. Así, MadSci ofrece una página de bienvenida que invi- 
ta a los usuarios a que comprueben los recursos existentes y listas de tipos de cuestiones que el servicio no responde. De este modo, sólo el $48 \%$ de usuarios pasan a la página de formular la pregunta, de los cuales únicamente el $22 \%$ finalmente la llegan a cumplimentar. Se han basado en el paquete gratuito Glimpse que archiva información de preguntas y respuestas, respondiendo automáticamente a las que se encuentran archivadas.

\section{The Internet Public Li- brary.}

Ofrece 8 servicios esenciales: centro de referencia con servicio $M O O$, división joven que incluye un servicio listserv para literatura infantil, servicios para bibliotecarios, espacio para exposiciones, aula para clases a distancia, sala de lectura directorio que contiene todos sus recursos electrónicos y herramientas de búsqueda web.

http://ipl.sils.umich.edu/

El servicio $M O O$ se llama $\mathrm{Ipl}$ moo y la conexión puede hacerse por telnet a ipl.sils.umich.edu: 8888 pero la mejor opción es utilizar la interfaz de un software cliente como MUDDweller para Macintosh o MUDwin para Windows. Cuando el usuario visita la sección "Ask a question" primero se le invita a que consulte las FAQ's. También se le advierte de que la respuesta puede tardar algún tiempo, cómo realizar la consulta y qué ocurre una vez recibida.

Han desarrollado su propio software denominado Quirc. Es un servidor especializado en www, gestiona el correo electrónico, hace seguimiento de las consultas y facilita la comunicación usuario/bibliotecario y entre los propios bibliotecarios. El personal es voluntario y pasa por un periodo de formación. También existe una política de respuestas y una base de datos que registra y gestiona todas las transacciones.

\section{24/7.}

Se inició como un proyecto gestionado por la California State Library y actualmente es un conjunto de herramientas de software que facilitan ayuda de referencia en tiempo real. Su misión es "proporcionar a las bibliotecas de las herramientas necesarias para llevar a cabo servicio de referencia en directo en la web". Algunas de sus funcionalidades son: chateo en tiempo real, navegación compartida, envío de ficheros, imágenes, presentaciones de Power Point, etc. Permite elaborar mensajes preconfigurados, transferir las preguntas a otros expertos y organizar las transcripciones por materia, idioma, categoría o cualquier otra clasificación que se necesite. Finalmente ofrece estadísticas e informes de uso.

http://www.24/ref.org/products.htm

\section{The Opal Project.}

Proyecto de la Open University (Reino Unido) de servicio 24 horas a sus 200.000 estudiantes a distancia. Otras dos universidades han participado también en el proyecto: University of Leicester Library Distance Learning Unit y la University of London's Birkbeck College Library. Con la creación de OpenLibr@ry, un portal para información en línea, han ido surgiendo numerosas cuestiones que deben ser tratadas por personal referencista. Para solucionar esta situación se crea Opal.

http://oulibl.open.ac.uk/wh/resear ch/opall

http://www.le.ac.uk/li/distance/ind ex.htm

http://www.bbk.ac.uk/lib/index.html http://oulib1.open.ac.uk/openlibra ry/index.htm

El objetivo final es diseñar un sistema propio a la medida para dar respuesta a estas cuestiones repetitivas y rutinarias. Como segundo objetivo se ha fijado la creación de un agente de inteligencia artificial. http://oulibl.open.ac.uk/wh/resear ch/opal/artificial.html

Otros servicios similares son los siguientes:

-Elite Project. Es un servicio de chat con HumanClick a través de un cuestionario web cerrado. http://www.le.ac.uk/li/distancelelit eproject/index.htm

-Ask a Librarian de las bibliotecas públicas británicas. Servicio de referencia por correo electrónico que comenzó en 1998. No responden a preguntas de fuera del país.

http://www.earl.org.uk/ask/index.h tml

-Remote Referencing HelpDesk Project, University of Edinburgh. Servicio de referencia de chat que asiste a los usuarios en su búsqueda en bases de datos.

http://www.remote.lib.ed.ac.uk/

- Macquarie University Library. Servicio de referencia digital basado en correo electrónico y conferencias. Utiliza el software de Microsoft NetMeeting, que permite videoconferencias, envío de mensajes de texto y pizarra compartida. http://www.lib.mq.edu.au/ask/

En cuanto a las propuestas en España cabe destacar las siguientes:

$-U O C$. Entre otros, ofrece un servicio de difusión selectiva de la información de acuerdo con un perfil personalizado del usuario. También tiene un boletín de sumario de revistas y de novedades de diarios económicos y de publicaciones oficiales.

http://www.uoc.es

- La biblioteca responde de la Biblioteca de Andalucía. Se trata de un servicio de referencia virtual vía correo electrónico. Se responde a preguntas concretas sobre Andalucía en un plazo máximo de 3 días hábiles.

http://www.sba.junta-andalucia. es/bgandalucia/servicios/referenci 


\section{aibibliografica/bibliotecaresponde .php}

-Universidad de Alicante. Se trata de un formulario web para cualquier tipo de consulta o solicitud a la biblioteca.

http://www.ua.es/es/bibliotecas/ser $v$ mformularios/index.htm

- Biblioteca Virtual Miguel de Cervantes. Otro formulario web para ayudar al usuario a encontrar recursos, resolver problemas técni$\cos$, información sobre la biblioteca, etc.

http://cervantesvirtual.com/bibliot ecariol

_Sai@Exe. De la Universidad de Jaén. Es un servicio de alerta informativa y distribución de sumarios de publicaciones electrónicas. http://www.ujaen.es/serv/biblio/ser vicios/sad/sai/index_sai.htm

-Pregunte? Las bibliotecas responden. Servicio de referencia digital basado en correo electrónico con 3 días de tiempo máximo de respuesta.

http://pregunte.carm.es/pregunte/

\section{Bibliografía}

Ammentorp, S.; Hummelshoj, M. "Ask a librarian: web-based reference question services. A model for development". En: $11^{\text {th }}$ NI\&D Conference, 2001.

http://www.bokis.is/iod2001/papers/Ammentorp paper.doc

Basic chat technologies. Consultado en: 25-0601.

http://pages.prodigy.net/tabol/basicchat.htm

Borchardt, K.; Croud, J. "Digital reference service: a new service, or the next logical step?". En: Educause in Australasia, 2001.

http://www.library.uq.edu.au/papers/digitalref.doc

Boyer, J. "Virtual reference at the Ncsu libraries: the first one hundred days". En: Information technology and libraries, 2001, v. 20, n. 3. http://www.lita.org/ital/2003_boyer.html

Breeding, M. "Providing virtual reference service". En: Information today, 1997, v. 8, n. 3, pp. $38-45$.

Broughton, K. "Our experiment in online, real-time reference". En: Computers in libraries, 2001, v. 21, n. 4, pp. 26-29.

http://www.infotoday.com/cilmag/apr01/brough ton.htm

Bry, L. Setting up an ask-an-expert service. Consultado en: 10-06-02.

http://www.madsci.org/ask_expert/
Butler, B. KnowledgeBit: a database format for reference version 2.0. Consultado en: 2106-02.

http://www.vrd.org/conferences/VRD2000/proc eedings/butler5-01.shtml

Coffman, S. "We'll take it from here: developments we'd like to see in virtual reference software". En: Information technology and libraries, 2001, v. 20, n. 3.

http://www.lita.org/ital/2003_coffman.html

Digital reference. Consultado en: 10-06-02. http://pages.prodigy.net/tabol/digref.htm

D’Angelo, B. J.; Maid, B. M. "Service from a distance: the use of information technologies in support of off-campus students". En: Cause 98 , 1998.

http://www.educause.edu/ir/library/html/cnc98 47/cnc9847.html

Eustance, K. "Going my way? Beyond the web and the MOO in the library". Consultado en: 27-06-02.

http://farrer.riv.csu.edu.au/ keustace/research/g mw.html

Fishman, Diane L. "Managing the Virtual Reference Desk: how to plan an effective e-mail reference service". En: Medical reference services quaterly, 1998, v. 17, n. 1, pp. 1-10.

Folger, K. M. "The virtual librarian: using desktop videoconferencing to provide interactive reference assistance". En: Acrl 1997 National Conference Papers.

http://www.ala.org/acrl/paperhtm/a09.html

Guidelines for information specialists of $K-12$ digital reference services. Consultado en: 1006-02.

http://www.vrd.org/training/guide.shtml

Hattery, M. "Live at ALA Midwinter: LC and your local public library". En: Information retrieval and library automation, 2001, v. 36, n. 9, pp. 1-3.

Horn, J. "The future is now: reference service for the electronic era". En: Acrl Tenth National Conference, 2001, pp. 320-327.

Kasowitz, A. S. Trends and issues in digital reference service. Consultado en: 21-06-02. http://www.ericit.org/digests/EDO-IR-200107.shtml

Lankes, R. D.; Collins, J. W.; Kasowitz, A. S. "Digital reference service in the new millenium: planning, management and evaluation" New York: Neal-Schuman Publishers, 2000. Isbn 1-5557-0384-4.

"LC, Oclc to collaborate in digital reference project". En: Advanced technology libraries, 2001, v. 30, n. 2, pp. 1-11.

Lessick, S.; Kjaer, K.; Clancy, S. "Interactive Reference Service (IRS) at UC Irvine: expanding reference service beyond the reference desk". En: Acrl 1997 National conference papers.

http://www.ala.org/acrl/paperhtm/a10.html

LiveRef(sm): a registry of real-time digital reference services. Consultado en: 11-06-02. http://www.public.iastate.edu/ CYBERSTACKS LiveRef.htm
McClennen, M.; Memmott, P. "Roles in digital reference". En: Information technology and libraries, 2001, v. 20, n. 3.

http://www.lita.org/ital/2003_mcclennan.html/

Merlo Vega, J. A. "El servicio bibliotecario de referencia". En: Anales de documentación, 2000, n. 3, pp. 93-126.

Oder, N. "The shape of e-reference". En: $\mathrm{Li}$ brary journal, 2001, v. 126, n. 2, pp. 46-50.

Osuna Alarcón, R. "Gestión y evaluación de un servicio de referencia e información bibliográfica". En: X Jornadas bibliotecarias de Andalucía, 1998, pp. 127-137.

Philip, B. An examination of the past, present and future of electronic mail reference service. Consultado en: 25-06-02.

http://www.slis.ualberta.ca/598/brenda/emailref .htm

Pinto Escribano, J. "Biblioteca virtual y usuarios remotos". En: Aabadom, 1997, v. 8, n. 3, pp. 38-45.

Ready for reference: Lsta grant guidelines for service provision. Consultado en: 21-06-02. http://www.alliancelibrarysystem.com/projects/ readyref/rrrg.htm

Riera Barsallo, P. "Servicios de información a medida para una comunidad virtual". En: XI Jornadas bibliotecarias de Andalucía, 2000, pp. 275-285.

Rollán Malmierca, A. "El servicio de referencia y el bibliotecario referencista". En: Boletín de la Asociación Andaluza de Bibliotecarios, 1998, v. 13, n. 51, pp. 9-26.

Ryan, S. "Reference service for the internet community: a case study of the internet public library reference". En: Library and information science research, 1996, v. 18, n. 3, pp. 241259.

Sears, J. "Chat reference service: an analysis of one semester's data". En: Issues in science and technology librarianship, 2001, n. 32. http://www.istl.org/istl/01-fall/article2.html

Sloan, B. Electronic reference service: some suggested guidelines. Consultado en: 21-06-02. http://alexia.lis.uiuc.edu/ b-sloan/guide.html

Sloan, B. Digital reference services bibliography. Consultado en: 25-03-02. http://alexia.lis.uiuc.edu/ b-sloan/digiref.html

Wasik, J. M. Building and maintaining digital reference services. Consultado en: 25-06-02. http://ericit.org/digests/EDO-IR-1999-04.shtml

Wasik, J. M. A report of library-related AskA services. Consultado en: 25-06-02. http://www.vrd.org/AskA/library.shtml

White, D. M. "Diffusion of an innovation: digital reference service in Carnegie Foundation master's". En: Journal of academic librarianship, 2001, v. 27, n. 3, pp. 173-187.

Purificación García Delgado, Hemeroteca, Universidad Politécnica de Valencia.

pgarciad@bib.upv.es 